\title{
X-RAY VARIABILITY IN V444 CYGNI: EVIDENCE FOR COLLIDING WINDS
}

\author{
M.F. CORCORAN ${ }^{1}$, I.R. STEVENS ${ }^{2}$, A.M.T. POLLOCK ${ }^{3}$, \\ J.H. SWANK ${ }^{4}$, S.N. SHORE ${ }^{5}$ and G.L. RAWLEY 6 \\ ${ }^{1}$ Universities Space Research Association, Code 668, \\ NASA/Goddard Space Flight Center, Greenbelt, MD 20771, U.S.A. \\ ${ }^{2}$ Institute of Astronomy, University of Cambridge, \\ Madingley Road, Cambridge CB3 OHA, U.K. \\ ${ }^{3}$ Computer \& Scientific Co., Ltd., 34 Westwood Rd., Sheffield S11 7EY, U.K. \\ ${ }^{4}$ Laboratory for High Energy Astrophysics, Code 666, \\ NASA/Goddard Space Flight Center, Greenbelt, MD 20771, U.S.A. \\ ${ }^{5}$ Department of Physics and Astronomy, Indiana University at South Bend \\ 1700 Mishawaka Avenue, South Bend, IN 46634, U.S.A \\ ${ }^{6}$ Applied Research Corporation, 8201 Corporate Dr., Landover, MD 20785, U.S.A.
}

\begin{abstract}
Phase-resolved observations of the soft X-ray flux from the WN5+O6 binary V444 Cyg (WR139) have been obtained by ROSAT. The objective of these observations were: (1) to compare our results with previous observations made with the IPC (Moffat et al. 1982); (2) to explore the phase dependence of the X-ray variations; and (3) to look for evidence of the generation of X-rays from colliding winds in the system. ROSAT observed V444 Cygni with the PSPC five separate times, for a total integration time of about $20 \mathrm{ksec}$. Figure 1 shows the phase-binned light-curve along with archival IPC data. The observed X-ray variability can be modeled as a wind eclipse of an X-ray source by the WR wind. If most of the X-rays from the system are produced in a shock-heated region of wind interaction between the two stars, then the shocked gas has a large physical extent $\left(r \approx 140 R_{W R}\right)$ if the observed variability is produced solely by the eclipse of the region by the WR wind. However, since the $O$ star makes a significant contribution to the total $\mathrm{X}$-ray flux from the system, the actual size of the shocked interaction region is probably much smaller.
\end{abstract}

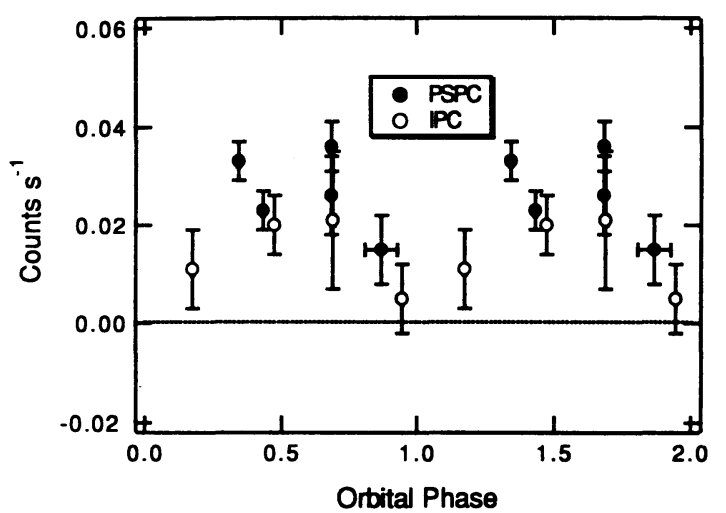

Fig. 1. The PSPC and IPC X-ray light-curve of V444 Cygni

\section{References}

Moffat, A.F.J., Firmani, C., McLean, I.S., Seggewiss, W. 1982, in: C.W.H. de Loore \& A. Willis (eds.), Wolf-Rayet Stars: Observations, Physics, Evolution, Proc. IAU Symp. No. 99 (Dordrecht: Reidel), p. 577 